14

\title{
Динамика накопления протопорфирина IX, индуцированного 5-аминолевулиновой кислотой в трех клеточных линиях разного происхождения
}

\author{
(C) Д.А. Горбенко, ${ }^{1,2}$ А.В. Белашов, ${ }^{1}$ Т.Н. Беляева, ${ }^{3}$ Е.С. Корнилова, ${ }^{3,4}$ И.К. Литвинов, ${ }^{3}$ \\ И.В. Семенова, ${ }^{1}$ О.С. Васютинский ${ }^{1}$ \\ ${ }^{1}$ Физико-технический институт им. А.Ф. Иофффе РАН, \\ 194021 Санкт-Петербург, Россия \\ 2 Университет ИТМО, \\ 197101 Санкт-Петербург, Россия \\ ${ }^{3}$ Институт цитологии РАН, \\ 194064 Санкт-Петербург, Россия \\ ${ }^{4}$ Санкт-Петербургский политехнический университет Петра Великого, \\ 195251 Санкт-Петербург, Россия \\ e-mail: daryarogova7@gmail.com
}

Поступило в Редакцию 23 апреля 2020 г. В окончательной редакции 2 июля 2020 г. Принято к публикации 6 июля 2020 г.

С помощью конфокальной флуоресцентной микроскопии исследована динамика синтеза и накопления протопорфирина IX, индуцированного 5-аминолевулиновой кислотой (5-ALA) в трех постоянных клеточных линиях: в иммортализованных клетках, полученных из опухолевых тканей человека - эпителиальной карциномы HeLa и аденокарциномы легкого A549, и фибробластоподобных иммортализованных клетках эмбрионов мыши 3T3. Накопление протопорфирина IX в клеточных культурах оценивалось на основе анализа интенсивности его флуоресценции. Исследована динамика изменения интенсивности флуоресценции в зависимости от времени инкубации клеток с 5-ALA и ее концентрации.

Ключевые слова: фотодинамическая терапия, протопорфирин IX, 5-аминолевулиновая кислота, конфокальная флуоресцентная микроскопия.

DOI: $10.21883 / J T F .2021 .01 .50288 .147-20$

Фотодинамическая терапия (ФДТ) находит широкое применение в современной онкологии для лечения широкого круга злокачественных новообразований. Основой ее успешного использования является генерация активных форм кислорода с помощью фотосенсибилизаторов (ФС) - веществ синтетического и природного происхождения. Накопление ФС в клетках злокачественных опухолей и генерация ими активных форм кислорода под воздействием оптического излучения приводит к запуску механизмов клеточной гибели, причем разные дозы облучения могут приводить к преобладанию определенного механизма гибели клеток, а также к разной скорости ее протекания $[1,2]$. Более того, для клеток различных локализаций опухоли разные механизмы гибели могут реализовываться при существенно отличающихся дозах облучения [1]. ФС активно используют и в диагностических целях для определения локализации опухоли, трофических язв и некоторых других патологий, а также для оценки размеров повреждений и исследования их морфологии [3]. К настоящему времени разработаны и используются в клинической практике ФС разных типов: хлорины, порфирины, фталоцианины, а также 5-аминолевулиновая кислота (5-ALA). Как известно, 5-ALA является предшественником эндогенно образующегося протопорфирина IX (PpIX) [4], который, в свою очередь, является важным прекурсором миоглобина, цитохрома и гема [5]. Было показано, что PpIX синтезируется в митохондриях клеток и проявляет свойства фотосенсибилизатора [6,7], поэтому подробная информация о его генерации в клетках опухолей различных локализаций имеет большое значение для применения в ФДТ и диагностике.

В некоторых типах клеток и тканей скорость синтеза РрIX определяется скоростью синтеза эндогенной 5-ALA. Синтез 5-ALA регулируется механизмом контроля с обратной связью. Работу данного механизма обеспечивают определенные концентрации свободного гема. Присутствие экзогенной 5-ALA обходит механизм контроля обратной связи и, таким образом, может индуцировать внутриклеточное накопление повышенных концентраций $\mathrm{PpIX}$, однако это происходит только в определенных типах клеток и тканей. Известно $([7,8])$, что 5-ALA более интенсивно накапливается в опухолевой ткани, что приводит к более активной генерации PpIX. Такая тканеспецифическая фотосенсибилизация обеспечивает основу для использования 5-ALA-индуцированного PpIX для ФДТ и диагностики [9]. Поэтому представляет интерес изучение эффективности генера- 
ции и аккумуляции PpIX в разных клеточных линиях, а также сравнение с его синтезом в незлокачественных клетках. Ранее механизмы генерации и внутриклеточной локализации PpIX были изучены в клеточных линиях карциномы гортани, рака печени, эмбриональной почки человека [6], глиобластомы [10], медуллобластомы [11] и рака кожи [12].

В настоящей работе проведено исследование динамики 5-ALA-индуцированного накопления PpIX в клетках трех клеточных линий: карциномы шейки матки человека (HeLa), карциномы легкого человека (A549), и фибробластоподобных иммортализованных клетках эмбрионов мыши (3Т3). Выбор клеточных линий определялся разной степенью их канцерогенности. Так, наиболее канцерогенными из выбранных трех типов считаются клетки линии А549, в то время как клетки типа 3Т3 являются псевдонормальными, т.е. они иммортализованы, но сохраняют свойства нормальных клеток. Необходимо отметить, что несмотря на то, что клетки HeLa и A549 - это клетки человека, а 3Т3 - клетки мыши, все три клеточные линии являются стандартными моделями для цитологических исследований и позволяют проводить сопоставления.

Все клеточные образцы культивировали в чашках Петри на стеклах в среде DMEM, содержащей 10\% эмбриональной бычьей сыворотки и $1 \%$ пенициллина/стрептомицина в атмосфере $5 \% \mathrm{CO}_{2}, 50 \%$ влажности при $37^{\circ} \mathrm{C}$. Эксперименты проводили через $48 \mathrm{~h}$ после посева клеток при достижении 60-70\% монослоя. Далее культуральную среду заменяли на среду, содержащую 5ALA в нужной концентрации, и клетки инкубировали указанное время.

Первая часть настоящей работы была посвящена определению времени инкубации клеток в растворе, содержащем 5-ALA, приводящему к наиболее эффективной генерации PpIX. Также изучалась зависимость от концентрации 5-ALA, вносимой в культуральную среду. Клетки линии HeLa инкубировали в течение 1, 2, 3 и $4 \mathrm{~h}$ при четырех концентрациях 5-ALA в культуральной среде: $0,50,100$ и $200 \mu \mathrm{g} / \mathrm{mL}$. После этого клетки промывали фосфатным буфером PBS и исследовали на конфокальном флуоресцентном микроскопе Olympus FV3000. Флуоресценцию PрIX возбуждали на длине волны 405 $\mathrm{nm}$ и детектировали в диапазоне длин волн 610-650 nm. Для получения устойчивого сигнала от протопорфирина, квантовый выход флуоресценции которого оказывается достаточно низким по сравнению с сигналами искусственных флуорофоров, синтезированных специально для использования в флуоресцентной микроскопии, был использован максимальный коэффициент усиления ФЭУ в флуоресцентном микроскопе. При этом сравнительно низкая плотность мощности возбуждающего лазера позволила избежать быстрого повреждения клеток, вызванного фотосенсибилизированной генерацией активных форм кислорода. Для обеспечения корректного сравнения данных о накоплении PpIX в разных типах клеток, а также при разной концентрации 5-ALA и разном времени инкубации, параметры чувствительности ФЭУ и время экспозиции поддерживались неизменными в ходе всего эксперимента. Одним из важных преимуществ конфокальной флуоресцентной микроскопии в данном случае является возможность регистрации достаточно слабого сигнала от PpIX, обусловленного незначительным квантовым выходом его флуоресценции.

Трехмерные распределения интенсивности флуоресценции $\mathrm{PpIX}$ регистрировались в нескольких полях в каждом образце, сканирование образцов проводилось с шагом $1 \mu \mathrm{m}$ по оси $Z$. Анализ полной интенсивности флуоресценции в клетках проводился с использованием программного обеспечения ImageJ [13]. Для измерения полного сигнала флуоресценции со всей клетки производилось суммирование флуоресцентных изображений в различных поперечных сечениях, регистрируемых в конфокальном флуоресцентном микроскопе. Впоследствии производилась сегментация отдельных клеток, предполагающая выделение их примерных границ на регистрируемых изображениях, полученных в режиме „на пропускание“. Это позволяло однозначно задать выделяемые области и верно разделить смежные клетки. Для учета возможного влияния размеров отдельных клеток на интегральный сигнал флуоресценции, суммарный сигнал флуоресценции, полученный описанным выше образом, делился на площадь клетки, определяемой в ходе ее сегментации. Это позволяло определить величину средней интенсивности флуоресценции в клетке $\overline{I_{t o t}}$.

Из-за возможного наличия в полученном сигнале помех, источниками которых являются в том числе темновой шум ФЭУ и флуоресценция самой питательной среды, для определения интенсивности флуоресценции, обусловленной только протопорфирином IX, накопленным внутри клеток, при каждом измерении учитывалась также средняя интенсивность фоновой области $I_{b g}$, в которой клетки отсутствовали. Искомая интенсивность флуоресценции PpIX (I $\mathrm{PpIX})$ в клетках определялась как разность полученной интегральной интенсивности флуоресценции клетки $\left(\overline{I_{t o t}}\right)$ и интегральной интенсивности флуоресценции фона $\left(I_{b g}\right)$ - области внеклеточной среды такого же объема:

$$
I_{\text {int }}=I_{t o t}-I_{b g}
$$

Полученные таким образом результаты по 50 отдельным клеткам в каждом образце анализировались статистически, определялось среднее значение интенсивности флуоресценции PpIX и его среднеквадратичное отклонение.

Интенсивность флуоресценции PpIX была проанализирована как функция времени инкубации с 5-ALA и концентрации 5-ALA. Было продемонстрировано, что максимальное накопление PpIX происходит после 3-часовой инкубации в растворе, содержащем 5-ALA в концентрации $100 \mu \mathrm{g} / \mathrm{mL}$. При этом важно отметить, что увеличение концентрации 5-ALA со 100 до $200 \mu \mathrm{g} / \mathrm{mL}$ 


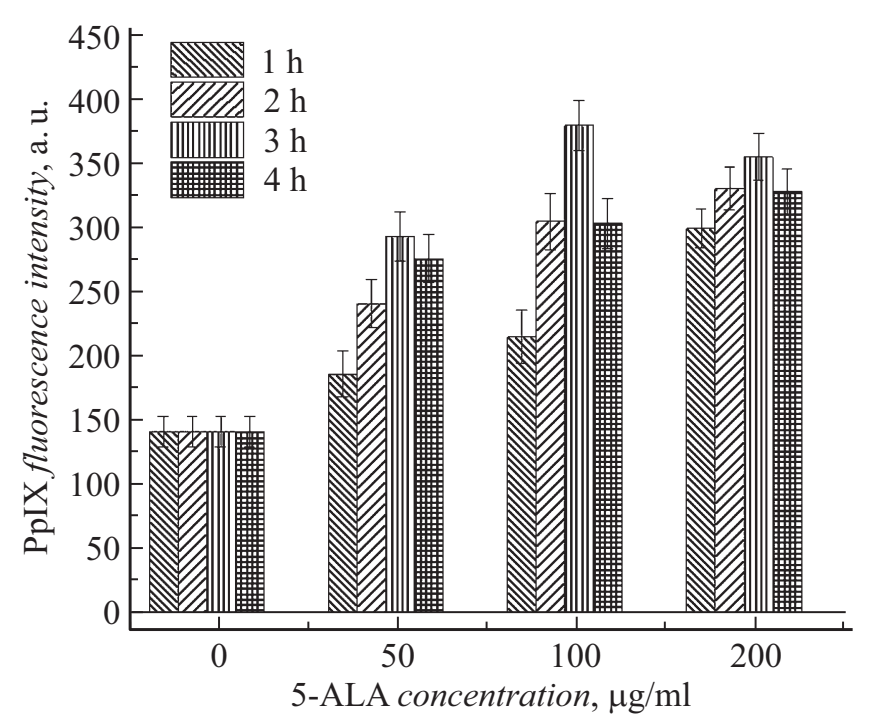

Рис. 1. Интенсивность флуоресценции PpIX в клетках линии HeLa в зависимости от времени инкубации при разных концентрациях 5-ALA.

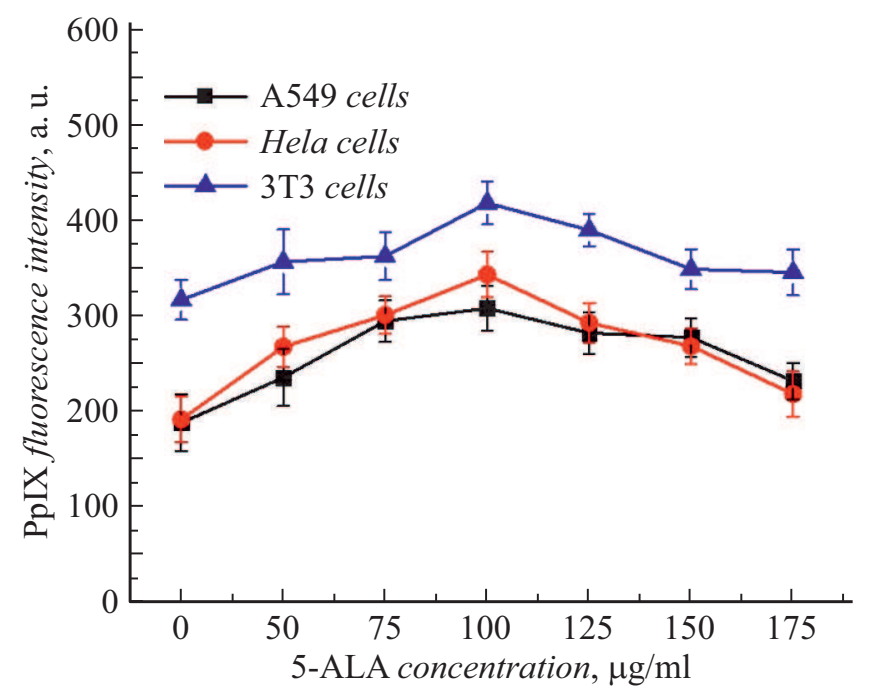

Рис. 2. Интенсивность флуоресценции РрIX в исследуемых клеточных линиях как функция концентрации 5-ALA. Указанные погрешности измерений соответствуют доверительной вероятности 0.95 по Стьюденту.

при 3-часовом культивировании не вызывало увеличения флуоресценции PpIX. На рис. 1 приведены гистограммы полной интенсивности флуоресценции PpIX в клетках HeLa в зависимости от концентрации 5-ALA и длительности накопления.

Вторая часть настоящей работы была посвящена анализу эффективности накопления $\mathrm{PpIX}$ в разных типах клеток в зависимости от концентрации 5-ALA в культуральной среде. Клетки HeLa, A549 и 3T3 инкубировали в течение $3 \mathrm{~h}$ при концентрациях 5-ALA в культуральной среде $0,50,75,100,125,150$ и $175 \mu \mathrm{g} / \mathrm{mL}$. Анализ интенсивности флуоресценции производился аналогично первой серии экспериментов, количественная оценка проводилась с помощью формулы (1). На рис. 2 приведена полученная зависимость интенсивности флуоресценции PpIX от концентрации 5-ALA для трех исследуемых клеточных линий. Как видно из рис. 2, концентрации 5-ALA выше $100 \mu \mathrm{g} / \mathrm{mL}$ не обеспечивали увеличения накопления PpIX. Полученные зависимости накопления PpIX от концентрации 5-ALA отражают обычную кинетику ферментативного процесса, для которой характерна колоколообразная форма $([14,15])$. Аналогичные зависимости были получены другими авторами для других клеточных линий $([16,17])$. Также нужно принимать во внимание, что большие концентрации 5-ALA могут оказывать повреждающее действие на метаболическую активность клеток в целом. Таким образом, мы продемонстрировали, что максимальное накопление PpIX во всех трех клеточных линиях достигается при 3-часовой инкубации с 5-ALA в концентрации $100 \mu \mathrm{g} / \mathrm{mL}$.

На рис. 3 приведены репрезентативные флуоресцентные изображения клеток линий A549, HeLa и 3T3, инкубированных без 5-ALA $(a-c)$ и после 3-часовой инкубации с 5-ALA в концентрации $100 \mu \mathrm{g} / \mathrm{mL}(d-f)$. Как видно из рисунка, все исследуемые клеточные культуры демонстрируют флуоресцентный сигнал самостоятельно синтезированного эндогенного PpIX. Внутриклеточная локализация сигнала неоднородна и, по всей видимости, ассоциирована с определенными внутриклеточными структурами. В соответствии с литературными данными такими структурами могут быть митохондрии $([18,19])$.

Из полученных данных было определено относительное увеличение сигнала интегральной флуоресценции PpIX в клетках исследуемых линий. Как видно из рис. 2, максимальное увеличение сигнала флуоресценции PpIX в иммортализованных клетках, полученных из опухолевых тканей (HeLa и A549), в сравнении с контрольными образцами без 5-ALA в культуральной среде составляет в среднем 63.2\%. В иммортализованных клетках нормальных фибробластов мыши (линия 3Т3) это увеличение составляет только $30 \%$. При этом необходимо отметить, что флуоресцентный сигнал в клетках линии 3Т3 в контрольных образцах в среднем в 1.7 раз выше, чем в клетках линий HeLa и A549, что говорит о более эффективном синтезе эндогенно образованного PрIX в иммортализованных клетках $3 \mathrm{~T} 3$.

Таким образом, в результате работы были определены оптимальная концентрация 5-ALA и время инкубации клеток in vitro, приводящие к наиболее эффективной генерации протопорфирина IX в трех постоянных иммортализованных клеточных линиях: двух, полученных из опухолевых тканей человека, и одной, полученной из нормальных фибробластов мыши. Показано, что оптимальные концентрации 5-ALA одинаковы для клеток разного происхождения. Полученные данные позволяют предполагать, что 5-ALA-индуцированное накопление 

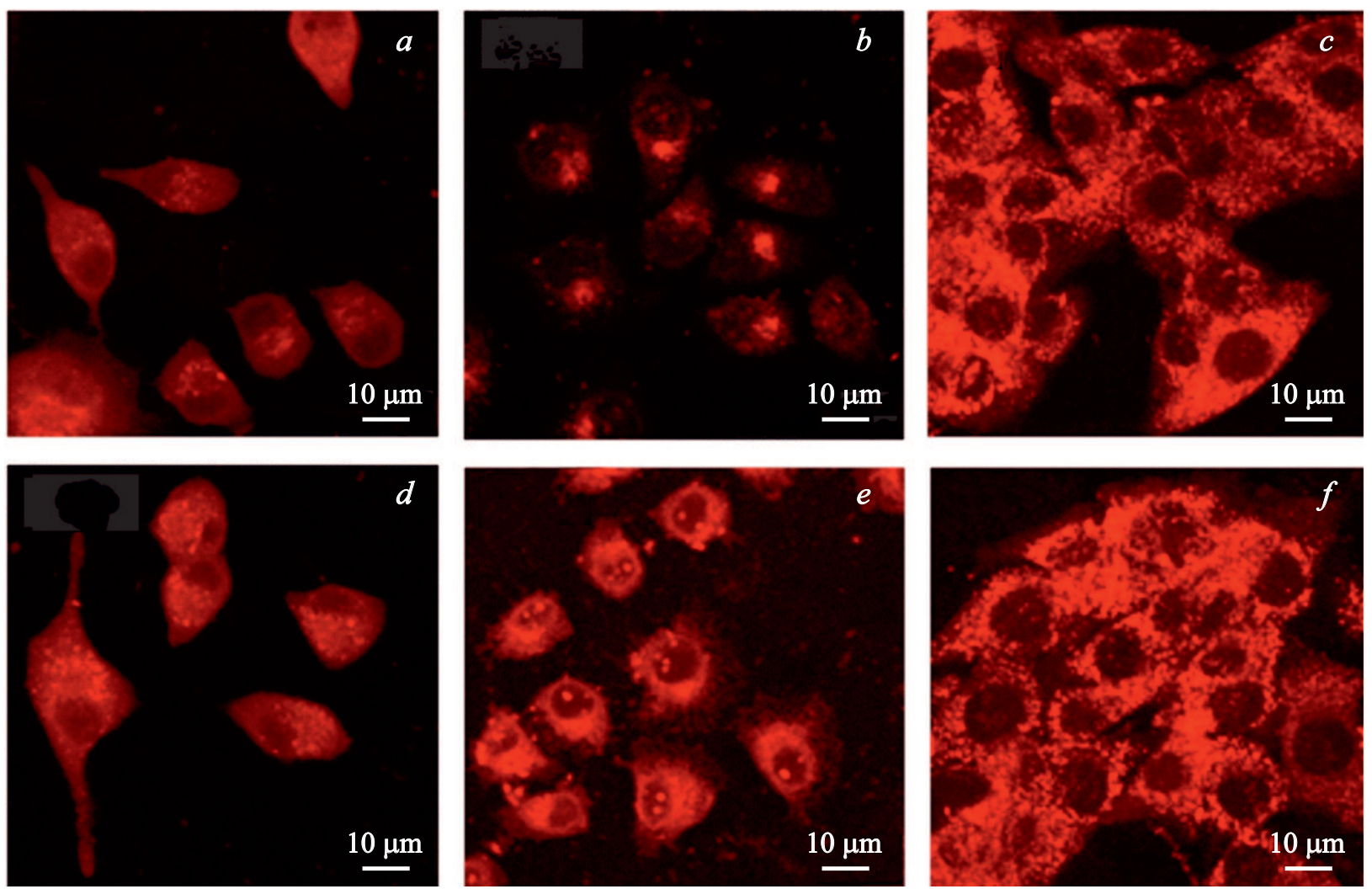

Рис. 3. Флуоресцентные изображения клеток $\mathrm{A} 549(a, d)$, HeLa $(b, e)$ и $3 \mathrm{~T} 3(c, f)$, инкубированных без 5-ALA (a-c) и после 3-часовой инкубации с 5-ALA в концентрации $100 \mu \mathrm{g} / \mathrm{mL}(d-f)$.

PpIX в клетках может быть связано со степенью злокачественности, однако не исключено, что этот эффект является тканеспецифичным.

\section{Благодарности}

А.В. Белашов благодарит Совет по грантам Президента Российской Федерации (Проект СП-2349.2019.4), а Т.Н. Беляева, И.К. Литвинов и Е.С. Корнилова - Министерство науки и высшего образования РФ (регистрационный номер темы АААА-А19-119020190093-9) за финансовую поддержку.

\section{Конфликт интересов}

Авторы заявляют, что у них нет конфликта интересов.

\section{Список литературы}

[1] A.A. Zhikhoreva, A.V. Belashov, D.A. Gorbenko, T.N. Belyaeva, E.S. Kornilova, I.V. Semenova, O.S. Vasyutinskii. Rus. J. Phys. Chem. B, 38 (6), 8 (2019).

[2] D.A. Gorbenko, A.V. Belashov, T.N. Belyaeva, E.S. Kornilova, I.V. Semenova, O.S. Vasyutinskii. JPCS, 1236 (1), 1 (2019).

[3] H. Abrahamse, M.R. Hamblin. Biochem. J., 473 (4), 347 (2016).
[4] J. Moan, O. Bech, J.M. Gaullier, T. Stokke, H.B. Steen, L.W. Ma, K. Berg. Int. J. Cancer., 75, 134 (1998).

[5] M. Wachowska, A. Muchowicz, M. Firczuk, M. Gabrysiak, M. Winiarska, M. Wańczyk, K. Bojarczuk, J. Golab. Molecules, 16, 4140 (2011).

[6] D.J. Piacquadio., D.M. Chen, H.F. Farber, J.F. Fowler Jr., S.D. Glazer, J.J. Goodman, L.L. Hruza, E.W. Jeffes, M.R. Ling, T.J. Phillips. Arch Dermatol., 140 (1), 41 (2004).

[7] K. Mahmoudi, K.L. Garvey, A. Bouras, G. Cramer, H. Stepp, J.G. Jesu Raj, D. Bozec, T.M. Busch, C.G. Hadjipanayis. J. Neurooncol. 141 (3), 595 (2019).

[8] S. Firdousa, M. Nawaza, M. Ikram, M. Ahmed. Biology and Medicine, 2 (3), 626 (2012).

[9] J.C. Kennedy, R.H. Pottier. J. Photochem. Photobiolog. B: Biology, 4 (4), 275 (1992).

[10] J.E. Lawrence, A.S. Patel, R.A. Rovin, R.J. Belton, C.E. Bammert, C.J. Steele, R.J. Winn. International Scholarly Research Notices 2014, 405360.

[11] A. Briel-Pumpa, T. Beez, L. Ebbert, M. Remkecde, S. Weinhold, M.C. Sabel, R.V. Sorga. J. Photochem. Photobiolog. B: Biology, 189, 298 (2018).

[12] K.R. Rollakanti, S.C. Kanick, S.C. Davis, B.W. Pogue, E.V. Maytin. Photon. Laser. Med., 2 (4), 287 (2013).

[13] P. Bankhead. Queen's University Belfast, Manual. (2014).

[14] K.A. Johnson. FEBS Lett., 587 (17), 2753 (2013).

[15] D.L. Purich. Enzyme Kinetics: Catalysis \& Control A Reference of Theory and Best-Practice Methods. (Elsevier, 2010), $920 \mathrm{p}$. 
[16] E. Gallegos, I. Rodríguez, L. Martínez Guzmán, A. Pérez Zapata. Archives of Medical Research, (30), 163 (1999).

[17] J.E. Lawrence, A.S. Patel, R.A. Rovin, R.J. Belton Jr., C.E. Bammert, C.J. Steele, R.J. Winn. ISRN Surgery, 2014, 1 (2014).

[18] Y. Lin, H. Chang, F. Chang, C. Shen. FEBS Lett., 587 (19), 3202 (2013).

[19] M. Sachar, K.E. Anderson, X. Ma. J Pharmacol Exp Ther., 356 (2), 267 (2016). 